\title{
Myasthenia Gravis and Thymectomy
}

\author{
V. Bharath ${ }^{1}$ (1) \\ ${ }^{1}$ Department of Cardiothoracic and Vascular Surgery, All India \\ Institute of Medical Sciences, New Delhi, India \\ J Card Crit Care 2021;5:229-237.
}

\author{
Abstract \\ Keywords \\ - myasthenia gravis \\ - thymectomy \\ - plasmapheresis \\ - IVIg \\ - VATS \\ - robotic thymectomy
}

\begin{abstract}
Address for correspondence Bharath V, MCh, Department of Cardiothoracic and Vascular Surgery, All India Institute of Medical Sciences, New Delhi, 110029, India (e-mail: drbharathv@gmail.com).
\end{abstract}

\section{Introduction}

Myasthenia gravis (MG) is a neuromuscular disorder characterized by weakness and fatigability of skeletal muscles. ${ }^{1}$

The first described case of MG is likely that of the Native American Chief Opechancanough, who died in 1664, as reported by Virginian chroniclers: "The excessive fatigue he encountered wrecked his constitution; his flesh became macerated; his sinews lost their tone and elasticity; and his eyelids were so heavy that he could not see unless they were lifted up by his attendants ... he was unable to walk; but his spirit rising above the ruins of his body directed from the litter on which he was carried by his Indians." ${ }^{2}$ In 1672, the English physician Thomas Willis described a patient with the "fatigable weakness" of limbs and bulbar muscles characteristic of MG. ${ }^{3}$

In the late 1800 s, the first modern descriptions of patients with myasthenic symptoms were published, and the name "myasthenia gravis" was coined by fusing the Greek terms for muscle and weakness to yield the noun "myasthenia" and adding the Latin adjective "gravis," which means severe. ${ }^{4}$

\section{Epidemiology}

MG can occur in any sex and at any age. Women are predominantly affected. A study ${ }^{5}$ reported that the women are affected nearly three times more often than men during early adulthood below 40 years of age.

Incidence is roughly equal during puberty and after the age of 40 years and is higher in men after 50 years of age. Overall incidence varies widely with place and population studied. A review study ${ }^{6}$ has reported incidence varying from 1.7 to 10.4 per million, and has been reported to be as high as 21 per million in Barcelona, Spain. A recent systematic review ${ }^{7}$ reported incidence rate from 1.7 to 21.3 cases per million person years. There is no epidemiological study till date from India.

Prevalence has increased over time, most likely to be due to the improved diagnosis and treatment of MG, and an increasing longevity of the population in general. As per recent study, ${ }^{8}$ prevalence has approached 20 per 100,000 in the US population. A meta-analysis ${ }^{7}$ has reported a prevalence rate of 15 to 179 per million. published online

January 16, 2022
DOI https://doi.org/

10.1055/s-0041-1739528. ISSN 2457-0206.
(C) 2022. Official Publication of The Simulation Society (TSS), accredited by International Society of Cardiovascular Ultrasound (ISCU). All rights reserved.

This is an open access article published by Thieme under the terms of the Creative Commons Attribution-NonDerivative-NonCommercial-License, permitting copying and reproduction so long as the original work is given appropriate credit. Contents may not be used for commercial purposes, or adapted, remixed, transformed or built upon. (https://creativecommons.org/ licenses/by-nc-nd/4.0/)

Thieme Medical and Scientific Publishers Pvt. Ltd., A-12, 2nd Floor, Sector 2, Noida-201301 UP, India 


\section{Pathophysiology}

The basic defect is decrease in the number of available acetylcholine receptors (AChRs) at neuromuscular junction due to antibody-mediated autoimmune response. Also, postsynaptic folds are flattened that cause decreased efficiency of neuromuscular transmission. Therefore, although acetylcholine $(\mathrm{ACh})$ is released normally, it produces small endplate potentials that may fail to trigger muscle action potentials.

The anti-AChR antibodies reduce the number of available AChRs at neuromuscular junction by three distinct mechanisms: (1) accelerated turnover of AChRs by a mechanism involving cross-linking and rapid endocytosis of the receptors; (2) damage to the postsynaptic muscle membrane by the antibody in collaboration with complement; and (3) blockade of the active site of the AChR, that is, the site that normally binds $\mathrm{ACh}{ }^{1}$

The postsynaptic transmembrane protein, muscle-specific tyrosine kinase (MuSK), is the main autoantigen in some MG patients. MuSK is a protein involved in AChR clustering at neuromuscular junction.

Autoimmune response in MG is not completely understood. The thymus appears to play a role in this process. The thymus is abnormal in $75 \%$ of patients with MG; in $65 \%$ the thymus is "hyperplastic," with the presence of active germinal centers detected histologically, though the hyperplastic thymus is not necessarily enlarged and an additional $10 \%$ of patients have thymic tumors (thymomas). ${ }^{1}$ There are muscle-like cells within the thymus called myoid cells that have AChRs on their surface. These may serve as a source of autoantigen and trigger the autoimmune reaction within the thymus gland.

Thymus pathology is common in MG patients, as first noted in the late 1800 s. $^{3}$ In 1937 , Blalock removed a mediastinal mass from a young woman who had $\mathrm{MG}^{3}$ the patient improved postoperatively. Later, Blalock et al reported other myasthenic patients who improved after thymus removal, ${ }^{9}$ establishing thymectomy as a treatment for MG.

In 1959 to 1960 , Nastuk et al proposed independently that MG has an autoimmune etiology. ${ }^{10}$

In 1973, Patrick and Lindstrom demonstrated that rabbits immunized with purified muscle-like AChR developed MGlike symptoms (experimental autoimmune $\mathrm{MG}$ ). ${ }^{11}$

\section{Clinical Features}

The clinical hallmark of MG is fatigable weakness, usually involving specific susceptible muscle groups. Patients often note fluctuating weakness from day to day or even from hour to hour, which worsens with activity and improves with rest or sleep. Patients can have varying degrees of ptosis, diplopia, dysarthria, dysphagia, dyspnea, facial weakness, or fatigable limb or axial weakness.

Ocular weakness presenting as fluctuating ptosis and/or diplopia is the most common initial presentation of MG $(85 \%){ }^{5}$ Bulbar weakness, presenting with painless dysphagia, dysarthria, or chewing difficulties, is the initial symptom in up to $15 \%$ of patients. Weakness involving respiratory muscles is rarely the presenting feature in first 2 years of onset.

The course of MG is often variable. Exacerbations and remissions may occur, particularly during the first few years after onset of disease. Disease progression to generalized weakness usually occurs within 2 years of disease onset. Weakness of facial muscles is quite common and many patients with MG have detectable weakness of eyelid closure with or without lower facial weakness when examined carefully, even when these muscle groups are not symptomatically weak. Spontaneous long-lasting remissions are uncommon, but have been reported in 10 to $20 \%$ of patients.

\section{Diagnosis}

The tests that are available to confirm the clinical diagnosis of MG include bedside tests, such as the edrophonium test, electrophysiological tests, and tests to measure the concentrations of serum autoantibodies.

\section{Bedside Tests}

The edrophonium test, which consists of administering edrophonium intravenously and observation of the patient for an improvement in muscle strength, can be most objectively and reliably interpreted with resolution of eyelid ptosis or improvement in strength of a single paretic extraocular muscle as the endpoints. Published reports indicate that its sensitivity in the diagnosis of MG is 71.5 to $95 \%$ for generalized disease.

\section{Electrophysiological Tests}

Repetitive nerve stimulation is the most commonly used electrophysiological test of neuromuscular transmission. In disorders of the NMJ, low rates of nerve stimulation $(2-5 \mathrm{~Hz})$ produce a progressive decrease or decrement in the amplitude of the compound muscle action potential. The result of the repetitive nerve stimulation test is abnormal in $~ 75 \%$ of patients with generalized MG ( $<50 \%$ of ocular MG), and is more likely to be abnormal in a proximal or facial muscle. single fiber electromyography (SFEMG) reveals abnormal jitter in 95 to $99 \%$ of patients with MG if appropriate muscles are examined.

\section{Immunological Tests}

The most commonly used immunological test for the diagnosis of MG measures the amount of serum antibody that precipitates muscle AChR. The sensitivity of this test is 85\% for generalized MG and 50\% for ocular MG. ${ }^{12}$ Patients with generalized MG who are anti-AChR negative should be tested for anti-MuSK antibodies, which are found in $~ 40 \%$ of patients in this group.

Striated muscle antibodies that recognize muscle cytoplasmic proteins (titin, myosin, actin, and ryanodine receptors) are not only detected in 75 to $85 \%$ of patients with thymomatous MG but also in some thymoma patients without MG. The presence of these antibodies in early-onset MG raises the suspicion of a thymoma. 


\section{Other Tests}

Chest computed tomography (CT) or magnetic resonance imaging (MRI) is done in all patients with confirmed MG to exclude the presence of a thymoma. Iodinated contrast agents should be used with caution because they might exacerbate myasthenic weakness. CT scan shows atrophic thymus in general adult population. Thymus may be normal, enlarged (hyperplastic), or may show a tumor (thymoma) in CT scan of patients with MG. It also gives information about thymus relationship with surrounding structures. In cases where it is difficult to distinguish between hyperplasia and thymoma on CT scan, MRI chest is useful. MRI distinguishes between hyperplasia and thymoma based on different signal intensity in various sequences.

MG often coexists with thyroid disease, so baseline testing of thyroid function should be obtained at the time of diagnosis. In anticipation of immunosuppressive treatment, screening for tuberculosis is desirable.

\section{Differential Diagnosis}

\section{Drug-Induced Myasthenia}

Treatment with penicillamine may result in true autoimmune MG, but the weakness is usually mild and recovery occurs within weeks or months after discontinuing its use. Aminoglycoside antibiotics or procainamide can cause exacerbation of weakness in myasthenic patients.

\section{Lambert-Eaton Myasthenic Syndrome}

Lambert-Eaton myasthenic syndrome (LEMS) is a presynaptic disorder of the neuromuscular junction that can cause weakness similar to that of MG. The proximal muscles of the lower limbs are most commonly affected, but other muscles may be involved as well. Cranial nerve findings, including ptosis of the eyelids and diplopia, occur in up to $70 \%$ of patients and resemble features of MG. However, the two conditions are usually readily distinguished, since patients with LEMS have depressed or absent reflexes and experience autonomic changes such as dry mouth and impotence. Nerve stimulation produces an initial low-amplitude response and at low rates of repetitive stimulation $(2-3 \mathrm{~Hz})$, decremental responses like those of MG occur; however, at high rates $(50 \mathrm{~Hz})$ or following exercise, incremental responses occur. LEMS is caused by autoantibodies directed against P/Q-type calcium channels at the motor nerve terminals, which can be detected in $85 \%$ of LEMS patients by radioimmunoassay. These autoantibodies result in impaired release of ACh from nerve terminals. Most patients with LEMS have an associated malignancy, most commonly small cell carcinoma of the lung, which may express calcium channels that stimulate the autoimmune response.

\section{Botulism}

Most commonly, botulism is caused by ingestion of improperly prepared food containing toxin. Rarely, the nearly ubiquitous spores of Clostridium botulinum may germinate in wounds. In infants, the spores may germinate in the gastrointestinal tract, and release toxin, causing muscle weakness.
Patients present with myasthenia-like bulbar weakness (e.g., diplopia, dysarthria, dysphagia) and lack sensory symptoms and signs. Weakness may generalize to the limbs and may result in respiratory failure. Reflexes are present early, but they may be diminished as the disease progresses. Mentation is normal. Autonomic findings include paralytic ileus, constipation, urinary retention, dilated or poorly reactive pupils, and dry mouth.

\section{Neurasthenia}

Neurasthenia is the historic term for a myasthenia-like fatigue syndrome without an organic basis. These patients may present with subjective symptoms of weakness and fatigue, but muscle testing usually reveals the "give-away weakness" characteristic of nonorganic disorders; the complaint of fatigue in these patients means tiredness or apathy rather than decreasing muscle power on repeated effort.

\section{Hyperthyroidism}

Hyperthyroidism is readily diagnosed or excluded by tests of thyroid function, which should be performed routinely in patients with suspected MG. Abnormalities of thyroid function (hyper or hypothyroidism) may increase myasthenic weakness.

\section{Progressive External Ophthalmoplegia}

Progressive external ophthalmoplegia is a rare condition resulting in weakness of the extraocular muscles, which may be accompanied by weakness of the proximal muscles of the limbs and other systemic features. Most patients with this condition have mitochondrial disorders that can be detected on muscle biopsy.

\section{Treatment}

MG is managed by medical therapy and surgical thymectomy.

\section{Medical Management}

This includes symptomatic therapy and immune therapy (short and long term).

\section{Symptomatic Therapy}

Attempts at rational treatments of MG began in the 1930s. A major step forward occurred in 1934 when Mary Walker realized that MG symptoms were similar to those of curare poisoning, which was treated with physostigmine, a cholinesterase inhibitor. She showed that physostigmine promptly improved myasthenic symptoms, ${ }^{11}$ making anticholinesterase drugs a staple in MG management.

Anticholinesterase medication produces at least partial improvement in most myasthenic patients, although improvement is complete in only a few. Pyridostigmine is the most widely used anticholinesterase drug. These are used in escalated doses and adjusted to maximize benefit and minimize side effects (diarrhea, stomach cramps). Pyridostigmine can be given 30 to 60 minutes before meals in patients with bulbar symptoms. Cholinesterase inhibitors rarely 
induce complete or sustained relief of MG symptoms. They do not affect disease progression, but might be sufficient for adequate management in certain patients with nonprogressive mild or purely ocular disease.

\section{Immune Therapy}

\section{Short-Term Immune Therapies}

Plasma exchange and intravenous immunoglobulin (IVIg)are used for short-term treatment of MG exacerbations. These are used when rapid clinical response is desirable.

Plasma exchange temporarily reduces the concentrations of circulating anti-AChR antibodies and produces improvement in a matter of days in most patients with acquired MG. Typically, one exchange, removing one to two plasma volumes, is done every other day, upto a total of four to six times. Published reports indicate that plasma exchange effectively improves strength in most patients with severe MG. ${ }^{13}$

IVIG is widely used for patients with exacerbating MG. Randomized controlled trials ${ }^{14}$ have shown similar efficacy of IVIG to plasma exchange in severe MG. The mechanisms by which IVIg produce improvement are not clear, but two important possibilities are competition with autoantibodies and Fc-receptor binding.

\section{Long-Term Immune Therapies}

They include the use of chronic immunosuppressive agents. This therapy is based on evidence from either small, randomized controlled trials, or anecdotal experience based on uncontrolled observations. The commonly used agents are corticosteroids, nonsteroidal immunosuppressants, and cytotoxic agents.

\section{Corticosteroids}

They are most commonly used first-line immunosuppressant. These are added when symptoms of MG are not adequately controlled by cholinesterase alone. They are started with high doses and gradually tapered off. Prednisolone is the most common steroid used. In four large retrospective series of steroid treatment for generalized MG, with steroid administered at various doses, more than $73 \%$ of the 422 patients treated achieved either marked improvement or remission. $^{15}$

\section{Nonsteroidal immunosuppressive agents}

a. Azathioprine is a purine antimetabolite that interferes with $\mathrm{T}$ cell and B cell proliferation. Retrospective studies indicate that azathioprine is effective in 70 to $90 \%$ of patients with MG, but the onset of benefit might be delayed for as long as 12 months. ${ }^{16}$ Azathioprine (initiated at $50 \mathrm{mg}$ daily) can be used alone or as a steroid-sparing agent in MG, but when used in combination with prednisone, it might be more effective and better tolerated than prednisone alone.

b. Mycophenolate mofetil blocks purine synthesis, thereby suppressing both $\mathrm{T}$ cell and $\mathrm{B}$ cell proliferation. Clinical efficacy in MG has been suggested by case series and in a retrospective study with MG. ${ }^{17}$ Two recently completed controlled trials of mycophenolate mofetil in MG failed to show additional benefit over $20 \mathrm{mg}$ daily prednisone given as initial immunotherapy or a significant steroid sparing effect in patients on steroid. $^{18}$

c. Cyclosporin inhibits $\mathrm{T}$ cell proliferation via disruption of calcineurin signaling, which blocks the synthesis of interleukin 2 and other proteins essential to the function of CD4 T cells. Its efficacy in MG has been suggested by randomized, placebo-controlled clinical trial. ${ }^{19} \mathrm{Few}$ retrospective studies have supported its use as a steroid-sparing agent. ${ }^{20}$ Cyclosporin is used mainly in patients in whom azathioprine is either ineffective or not tolerated.

d. Tacrolimus (FK506) has a similar mechanism of action as cyclosporin. Several reports have shown potential benefit in MG. A randomized unblinded study has shown similar results. ${ }^{21}$ Sustained benefit has been reported in antiryanodine-receptor positive patients, which has been hypothesized to be due to enhancement of ryanodine-receptor-related sarcoplasmic calcium release.

3. Other agents-A small percentage of patients with MG are refractory or develop intolerable side effects to treatment with corticosteroids in combination with one or more of the immunosuppressive agents described above. Agents that can be considered in these refractory patients include cyclophosphamide and rituximab.

a. Cyclophosphamide-In a recent randomized controlled trial, pulsed doses of intravenous cyclophosphamide $\left(500 \mathrm{mg} / \mathrm{m}^{2}\right)$ given to patients with refractory MG improved muscle strength and reduced steroid requirement. ${ }^{22}$ Remarkable therapeutic responses have also been reported in patients with refractory MG receiving a one-time, high-dose $(50 \mathrm{mg} / \mathrm{kg}$ ) intravenous course of cyclophosphamide for 4 days followed by rescue therapy, with benefit persisting for several years without relapse.

b. Rituximab-It is a chimeric monoclonal antibody directed against the $B$ cell surface marker CD20. It effectively reduces circulating $B$ cell counts and on the basis of its potential for targeting autoreactive B cell clones, it might have a therapeutic role in antibody-mediated autoimmune diseases. Several case reports have suggested benefit in patients with refractory MG and in those with MuSK MG. ${ }^{23}$ Further investigation is needed to determine its role in MG therapy.

MG is a chronic disease with remissions and relapses. Patients with MG require close follow-up care. During crisis, patients require mechanical ventilation and intensive care unit (ICU) care. Patients treated with medical management have remission rates (RRs) ranging from 6 to 34\% over a follow-up of 5 to 17 years. ${ }^{24}$ Since thymectomy studies report better RRs with increasing follow-up, surgical treatment may be considered in patients stabilized on medical treatment. 


\section{Surgical Treatment}

Surgical treatment of myasthenia consists of thymectomy.

\section{Perioperative Management and Anesthetic Considerations}

Patients with MG are stabilized with medical management by neurologist before taking up for surgery. Arterial blood gas and pulmonary function test are done to assess patient lung function to tolerate single lung ventilation during surgery. Incentive spirometry is started few weeks before surgery.

On the day of surgery, morning dose of steroids and immunosuppression medications is given. Patient is intubated with double lumen endotracheal tube if video-assisted thoracoscopic (VATS) thymectomy is planned. Arterial and central venous line are placed for monitoring. Midline sternotomy is done for open transsternal thymectomy and VATS ports are inserted for thoracoscopic thymectomy.

Postoperatively, patient is shifted to ICU or high dependency unit for monitoring. Medications are given through Ryle's tube. When patient becomes awake and gains adequate muscle power, extubation is done. Invasive lines and drains are removed on postoperative day 2. Patient is discharged on 4 th or 5 th day.

\section{Operative Techniques}

Abnormalities of thymus gland are common in patients of MG. Thymoma is present in 10 to $15 \%$ of patients and lymphoid follicular hyperplasia in $65 \%$ of patients. ${ }^{1}$ MG occurs in 30 to $35 \%$ of thymoma patients. ${ }^{25}$ Thymus plays an important immunopathological role in development of autoimmune MG.

The exact mechanism by which thymectomy produces benefits in MG is uncertain. The possible mechanism includes (1) thymectomy may eliminate source of continued antigen stimulation; (2) it may remove reservoir of B cells secreting AChR antibody; and (3) in some way it may correct a disturbance in immune regulation. ${ }^{26}$

The use of thymectomy in MG was initially based on empirical observations that patients with MG improved after removal of thymus. ${ }^{26}$ A comprehensive meta-analysis of 21 retrospective studies with 8490 patients suggested that patients having thymectomy were two times more likely to experience improvement than those who were unoperated. ${ }^{27}$ The improvement was defined as medication-free remission, asymptomatic on medication or improved on medication. However, the effect of thymectomy is usually not apparent until after 1 year, and full effect is not felt for 5 years. Based on above results, thymectomy is recommended in patients with nonthymomatous MG as an option to increase the probability of remission or improvement. The role of thymectomy in anti-MuSK MG is not clear. ${ }^{28}$ Thymomatous MG is an absolute indication for thymectomy.

There are various studies that have shown remission of symptoms in nonthymomatous and thymomatous MG following thymectomy. The first observation that thymectomy influenced course of MG was made by Sauerbruch et al, who in 1911 performed thymectomy on a girl with hyperthyroidism and $M G{ }^{29}$

Blalock et al first did thymectomy for a patient of MG in 1936 which had a truly catalytic effect on operative treatment of myasthenia. ${ }^{30}$ In 1954 , a series on thymectomy was published that showed improvement in two-thirds of the cases of MG after thymectomy. He also noticed that the results of thymectomy were better in patient with thymoma. $^{31}$

A retrospective review in 1976 from Mayo Clinic used computer-assisted matching to study effects of medical treatment and thymectomy in patient with non-thymomatous MG (age 17 to $>60$ years). The author reviewed 104 surgically treated patient and 459 medically treated patient and reported complete remission in $35 \%$ of surgical patients (at an average follow-up of 19.5 years) compared with $7.5 \%$ of the medical group (at an average follow-up of 23 years). ${ }^{32}$

A review of 375 cases of myasthenia done in 1996 who underwent extended thymectomy from 1973 to $1993 .{ }^{33}$ This included 98 patients of thymomatous MG and 286 patients of nonthymomatous MG. All of them underwent extended thymectomy with total resection of thymoma in thymomatous patients. Effects of extended thymectomy in above group were assessed separately by RR and palliation rate (PR). In nonthymomatous group, the RR were 36.9\% (3 years), $45.8 \%$ (5 years), $55.7 \%$ (10 years), and $67.2 \%$ (15 years). In thymomatous MG, the RR rose gradually, reaching $32.4 \%$ at 3 years and plateaued. PR in nonthymomatous MG reached $74 \%$ at 3 months, increased gradually until 3 years and plateaued over $90 \%$ thereafter and in thymomatous MG PR curve reached $82.5 \%$ at 1 year and plateaued.

Similar review of 662 patients of MG done in 1989 who underwent extended thymectomy over the span of 15 years consisting of 500 nonthymomatous MG patients and 162 thymomatous MG. ${ }^{34}$ This study reported remission in $37.9 \%$, improvement in $49.4 \%$, unchanged or worsened course in $7.4 \%$, and death in $5.2 \%$ of nonthymomatous MG patients. About $15.7 \%$ of thymomatous MG showed remission, $60.3 \%$ showed improvement, $3.7 \%$ remained unchanged or got worse, and rest of them (20.1\%) died.

A review was done by Emeryk and Strugalska in 150 cases of MG who underwent thymectomy. ${ }^{35}$ They followed 123 of 150 patients for 1 to 5 years after operation and reported remission in $24.4 \%$, significant improvement in $36.6 \%$, slight improvement in $24.4 \%$, and no improvement in $8.1 \%$ of cases, while deterioration occurred in $1.6 \%$ of patients.

A retrospective review ${ }^{36}$ of 100 patients of nonthymomatous myasthenia who underwent thymectomy between 1989 and 1998 were followed up for 5 years reported that $85 \%$ patient discharged on postoperative day 1 and $96 \%$ on postoperative day 2 . There was no intraoperative complication, but eight patients developed complication during postoperative stay (myasthenic crisis in one patient, deep venous thrombosis in one patient, five patient developed pneumothorax, and one patient had seizure) and no mortality within 30 days of surgery. The mean postoperative stay was 1.22 days (median: 0.72, range: 0.6-4.1). The median Osserman grade of all patients improved after surgery, that is, 3 to 1 
$(p<0.001)$. Eighty-five percent of patients improved one or more Osserman grades, $63 \%$ improved 2 or more grade, $14 \%$ of patients remained unchanged, and $1.3 \%$ patients deteriorated by one grade. Thirty-five percent of patients were in remission and $71 \%$ of them had no generalized symptoms.

A study included 478 patients with MG from 1973 to 1999, who underwent thymectomy through a partial median sternotomy reported no intraoperative or postoperative mortality. ${ }^{37}$ None of the patients required reoperation. There were 11 late deaths, none of them related to the operation. Among the causes of late death, five were attributed to a myasthenic crisis, two to mixed crisis, and four patients died due to causes unrelated to the disease. Four patients required ventilator support after operation and three of them were treated with plasmapheresis. Among the complications, one patient developed osteomyelitis in the sternal bone and five other patients had subcutaneous fluid collection. There were no phrenic nerve or inferior laryngeal nerve injuries, and there was no residual pneumothorax or hemothorax in this series of patients. Postoperative hospital stay ranged from 3 to 68 days with a mean of 7.3 days. Follow-up time ranged from 1 month to 25 years (mean: 7.3 years). Sixty-one patients (12.7\%) had complete remission of the symptoms, 299 (62.5\%) had a significant improvement, and 83 patients (17.4\%) had a mild improvement in the symptoms. Only 35 patients $(7.4 \%)$ did not show improvement in the baseline symptoms.

A retrospective study including 324 patients who underwent thymectomy from 1987 and 1996, through median sternotomy, investigated the postoperative complications. ${ }^{38}$ One-hundred forty-nine patients made an uneventful recovery; 104 patients had only minor complications, whereas 71 patients had major complications. The mortality rate was $0.6 \%$ ( 2 patients). The major surgical complications were recorded as sternal bleeding ( 1 patient) and sternal disruption (1 patient). The major general complications were recorded as tracheal stenosis (1 patient), pneumonia (3 patients), heart failure (1 patient), gastric hemorrhage (1 patient), and respiratory insufficiency (71 patients). Fortysix reintubations were performed on 40 patients and 19 tracheostomies (6\%) were performed postoperatively.

Shrager et al did a retrospective study that included 164 patients of MG who underwent transcervical thymectomy from 1992 to 2004 and studied the surgical and neurological outcome. ${ }^{39}$ They reported a complication rate of 7.3\%. Mean age at surgery was 43 years. Mean preoperative Osserman class was 2.3 (21\% in class $1,39 \%$ in class $2,28 \%$ in class $3,12 \%$ in class 4). Mean length of follow-up was 53 months. Mean postoperative Osserman class was 1 . The crude cumulative complete remission was $37 \%(n=58)$. Kaplan-Meier estimates of complete remission were 43 and $45 \%$ at 3 and 6 years, respectively. On multivariate analysis, only preoperative disease severity was significantly (inversely) associated with Kaplan-Meier complete remission rates (46\%).

The clinical outcome of 180 nonthymomatous MG who were surgically treated were investigated. ${ }^{40}$ The original surgical access consisted of a video-assisted inframammary cosmetic incision and median sternotomy. The medical record and the outpatients' clinic follow-up information of the 180 cases had been extensively reviewed. In addition to the strictly surgical benchmark referral, data on the rate of cure of the MG (complete stable remission-CSR; pharmacological remission -PR) as indicated by the Myasthenia Gravis Foundation of America (MGFA) have been analyzed as recorded at the 12 months after surgery checkpoint. Cosmetic outcome was evaluated as well. Female to male ratio was 156 (86.7\%):24 (13.3\%); mean age: $29.1 \pm 10.9$ years; preoperative MGFA score; stage I: 4 patients (2.2\%); Ila: 57 (31.7\%); IIb: 32 (17.8\%); IIIa: 41 (23.3\%); IIIb: 42 (23.3\%); IVa: 2(1.1\%); V: 2 (1.1\%). Median operative time was 110 minutes (70-130 minute) and median postoperative hospital stay was 4 days (3-10 days). Postoperative mortality was nil and morbidity occurred in seven patients (3.8\%). Final pathology was consistent with 146 hyperplastic thymus (81.1\%), 28 involuted thymus (15.6\%), and 6 normal thymus (3.3\%). Ectopic thymic tissue was found in $68 \%$ of the patients. Mean follow-up was $62.9 \pm 34.6$ months. A CSR was obtained in $55 \%$, PR in $18.3 \%$, improvement in $39.9 \%$, unchanged in $3.5 \%$, worse in $1.1 \%$, and died in $0.5 \%$. KaplanMeier estimates of CSR were 34.1 and $75.8 \%$ at 5 and 10 years, respectively. The preoperative therapy was the only parameter significantly associated with Kaplan-Meier CSR rates (univariate analysis $-p<0.001)$. Remarkably, $171(95 \%)$ patients judged their cosmetic results to be excellent or very good. Thymectomy in MG patients via video-assisted inframammary cosmetic incision and median sternotomy has shown to be a useful surgical approach as demonstrated by the good functional and very good aesthetic results, associated with a very low morbidity and no mortality. Patients with preoperative monotherapy have higher CSR rates. CSRs are durable, as the CSR rate improves with extended follow-up.

A study of 119 patients, aged between 12 and 83 years, who were surgically treated between 1998 and 2007 through video-assisted thoracoscopic approach, was reviewed for clinical outcome. ${ }^{41}$ Disease severity was graded using the Osserman classification. To prevent rupture of the tumor capsule and tumor seeding, thymomas were resected using a modified no-touch technique. Thymoma diameters were 10 to $90 \mathrm{~mm}$ (mean, $50 \mathrm{~mm}$ ). There were no operative deaths, 12 (10\%) patients had complications, and 87 (73.1\%) improved by 1 or more Osserman grades postoperatively. After followup of 1.9 to 10 years (mean, 4.9 years), 74 (62\%) patients remained asymptomatic, with $21 \%$ in CSR. Using multivariate regression analysis, there were no statistical differences in median pre- and postoperative Osserman grades with regard to age, sex, duration of symptoms, and presence of thymoma. Video-assisted thoracoscopic thymectomy for MG and selected thymomas can achieve long-term clinical outcomes comparable to those of standard approaches.

In a controlled study done by Buckingham et al, a 34\% remission and a 32\% improvement rate were achieved after thymectomy compared with 8 and $16 \%$ for matched patients without the operation ${ }^{32}$ (class III evidence).

Gronseth and Barohn asserted unequivocally that "for patients with non-thymomatous autoimmune Myasthenia 
gravis, thymectomy is recommended as an option to increase the probability of remission or improvement." Patients with MG undergoing thymectomy were twice as likely to attain medication-free remission, 1.6 times as likely to become asymptomatic and 1.7 times as likely to improve. No study found a significant negative influence of thymectomy. Patients with purely ocular manifestations did not benefit from thymectomy. The outcome for younger MG patients was not significantly different from the total MG group. Mild MG (Osserman grade 1-2) did not profit from surgery, while more severe cases (Osserman grade $2 \mathrm{~b}-4$ ) were 3.7 times as likely to achieve remission after thymectomy than those without surgery $(p<0.0077){ }^{27,42}$

Skeie et al stated that "Thymoma patients should have thymectomy. AChR antibody-positive early-onset patients with generalized Myasthenia gravis and insufficient response to pyridostigmine therapy should be considered for thymectomy, ideally within 1 year of disease onset." 43

Liu et al stated that "Thymectomy can achieve good longterm CSR in Myasthenia gravis, and VATS is an ideal alternative method. High-dose prescription of anti-cholinesterase and the advanced stage by Myasthenia Gravis Foundation of America (MGFA) classification have higher risks of postoperative crisis. Preoperative prescription of anti-cholinesterase alone and non-thymomatous Myasthenia gravis are good prognostic factors. Thymectomy should also be considered for the ocular type of Myasthenia

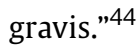

Tomulescu et al stated that "Outcomes of the thoracoscopic approach in MG without thymoma were similar to those provided by open surgery, with the acknowledged benefits of minimally invasive surgery and good patient acceptance. ${ }^{45}$

$\mathrm{Ng}$ et al stated that "VATS for thymectomy results in less postoperative pain, better preserved pulmonary function and improved cosmesis, which can be particularly important to many young female patients with myasthenia

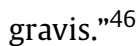

Yu et al stated that "Video-assisted thoracoscopic thymectomy can produce satisfactory long-term result." 47

Ponseti et al did a study to evaluate the role of ectopic thymic tissue in relation to clinical outcome in Spain. They studied 83 consecutive generalized seropositive nonthymomatous MG patients and found ectopic thymic tissue in 35 patients (42.2\%). Probability of obtaining CSR for groups without and with ectopic thymic tissue was 65 and 26\% at 5 years. They concluded that clinical outcome of patients with nonthymomatous seropositive MG is significantly affected by the presence of ectopic thymic tissue in the mediastinal fat. ${ }^{48}$

Ambrogi and Mineo also studied the role of ectopic thymic tissue in outcome following thymectomy in 51 patients. They concluded that presence of active ectopic thymus was the most significant predictor of poor outcome. ${ }^{49}$

Zahid et al stated that "VATS produces equivalent postoperative mortality and CSR, with many papers demonstrating superior results in terms of hospital stay, operative blood loss and patient satisfaction compared to the trans sternal approach. Hence, VATS may be considered in preference to the trans sternal approach despite its greater operative times." 50

A recent study from Mayo Clinic had 510 thymectomies done over 20 years with 56 done by minimally invasive techniques. This study concluded that VATS is associated with reduced blood loss, operative times, and earlier hospital discharge compared with robotic thymectomy. ${ }^{51}$

Liu et al retrospectively reviewed 103 consecutive patients who received VATET for nonthymomatous MG with minimal follow-up period of 36 months and concluded that the long-term outcome of right-sided VATET is comparable to that of bilateral VATET in terms of CSR. ${ }^{52}$

A systematic review conducted by Hess et al included 20 studies with 2,068 patients who underwent either MIT ( 838 patients) or open thymectomy (1,230 patients). They concluded that minimally invasive thymectomy (MIT) may reduce blood loss, chest tube duration, and hospital stay, with clinical outcomes comparable to open techniques in appropriately selected patients. ${ }^{53}$

The landmark randomized trial by Wolfe et al comparing thymectomy and steroids against only steroids in patients with nonthymomatous MG was published in 2016. ${ }^{54}$ It showed improved 3-year clinical outcomes in thymectomy group.

\section{Discussion}

Thymus is known to play an important role in the etiopathogenesis of MG and its surgical removal has been noted to improve outcomes in patients with MG. ${ }^{9,27}$ There are several approaches through which thymectomy can be done and there is difference of opinion with regard to the best surgical approach. Thymectomies can be done by transcervical, transsternal, or combined approach. ${ }^{55}$ Each approach differs with regard to the extent of thymic and ectopic thymic tissue resected as thymic tissue has been noted outside the confines of thymic capsule.

Thymectomy usually involves removal of thymus and fibrofatty tissue between the two phrenic nerves and from lower border of thyroid gland to the diaphragm. Traditionally, this has been done by trans sternal approach. With the advent of minimal invasive techniques, VATS has been used to perform thymectomy in patients with MG.

Minimally invasive thymectomy offers several advantages like low procedural morbidity, shorter hospital stays, better cosmesis, and reduced postoperative pain compared with trans sternal thymectomy.

\section{Conclusion}

The recent landmark randomized clinical trial has indicated improved clinical outcomes with thymectomy in comparison to medications alone. With the advent of minimal invasive thymectomy, the day in which patients with MG can be given longer remission is not far away.

Conflicts of Interest

None. 


\section{References}

1 Dan L, Kasper DL, Jameson JL, Fauci AS, Hauser SL, Loscalzo J. Harrison's Principles of Internal Medicine. 18th edition. New York, NY: The McGraw-Hill Companies; 2012, Chapter 386

2 Marsteller HB. The first American case of myasthenia gravis. Arch Neurol 1988;45(02):185-187

3 Pascuzzi RM. The history of myasthenia gravis. Neurol Clin 1994; 12(02):231-242

4 Keesey JC. "Crisis" in myasthenia gravis: an historical perspective. Muscle Nerve 2002;26(01):1-3

5 Grob D, Brunner N, Namba T, Pagala M. Lifetime course of myasthenia gravis. Muscle Nerve 2008;37(02):141-149

6 Phillips LH II, Torner JC. Epidemiologic evidence for a changing natural history of myasthenia gravis. Neurology 1996;47(05):1233-1238

7 Carr AS, Cardwell CR, McCarron PO, McConville J. A systematic review of population based epidemiological studies in Myasthenia Gravis. BMC Neurol 2010;10:46

8 Phillips LH II. The epidemiology of myasthenia gravis. Ann N Y Acad Sci 2003;998:407-412

9 Blalock A, et al. The treatment of myasthenia gravis by removal of the thymus gland. JAMA 1941;117:1529

10 Nastuk WL, Strauss AJ, Osserman KE. Search for a neuromuscular blocking agent in the blood of patients with myasthenia gravis. Am J Med 1959;26(03):394-409

11 Patrick J, Lindstrom J. Autoimmune response to acetylcholine receptor. Science 1973;180(4088):871-872

12 Lindstrom JM, Seybold ME, Lennon VA, Whittingham S, Duane DD. Antibody to acetylcholine receptor in myasthenia gravis. Prevalence, clinical correlates, and diagnostic value. Neurology 1976 ; 26(11):1054-1059

13 Newsom-Davis J, Pinching AJ, Vincent A, Wilson SG. Function of circulating antibody to acetylcholine receptor in myasthenia gravis: investigation by plasma exchange. Neurology 1978;28(03):266-272

14 Gajdos P, Chevret S, Clair B, Tranchant C, Chastang CMyasthenia Gravis Clinical Study Group. Clinical trial of plasma exchange and high-dose intravenous immunoglobulin in myasthenia gravis. Ann Neurol 1997;41(06):789-796

15 Pascuzzi RM, Coslett HB, Johns TR. Long-term corticosteroid treatment of myasthenia gravis: report of 116 patients. Ann Neurol 1984;15(03):291-298

16 Witte AS, Cornblath DR, Parry GJ, Lisak RP, Schatz NJ. Azathioprine in the treatment of myasthenia gravis. Ann Neurol 1984;15(06): 602-605

17 Chaudhry V, Cornblath DR, Griffin JW, O'Brien R, Drachman DB. Mycophenolate mofetil: a safe and promising immunosuppressant in neuromuscular diseases. Neurology 2001;56(01):94-96

18 Muscle Study Group. A trial of mycophenolate mofetil with prednisone as initial immunotherapy in myasthenia gravis. Neurology 2008;71(06):394-399

19 Tindall RS, Phillips JT, Rollins JA, Wells L, Hall K. A clinical therapeutic trial of cyclosporine in myasthenia gravis. Ann N Y Acad Sci 1993;681:539-551

20 Ciafaloni E, Nikhar NK, Massey JM, Sanders DB. Retrospective analysis of the use of cyclosporine in myasthenia gravis. Neurology 2000;55(03):448-450

21 Nagane Y, Utsugisawa K, Obara D, Kondoh R, Terayama Y. Efficacy of low-dose FK506 in the treatment of Myasthenia gravis-a randomized pilot study. Eur Neurol 2005;53(03):146-150

22 De Feo LG, Schottlender J, Martelli NA, Molfino NA. Use of intravenous pulsed cyclophosphamide in severe, generalized myasthenia gravis. Muscle Nerve 2002;26(01):31-36

23 Gardner R, Pestronk A, Al-Lozi MT. Intractable myasthenia gravis responding to rituximab treatment. Neurology 2008;70(Suppl 1): A301

24 Scadding GK, Havard CWH, Lange MJ, Domb I. The long term experience of thymectomy for myasthenia gravis. J Neurol Neurosurg Psychiatry 1985;48(05):401-406
25 Rosenberg JC. Neoplasm of Mediastinum. In: De Vita VT Jr., Hellman S, Rosenberg SA, eds. "Cancer Principles and Practice of Oncology.”. Philadelphia: J.B. Lippincott Company; 1905: 599-620

26 Blalock A, Harvey AM, Ford FF, Lilienthal J Jr. The treatment of myasthenia gravis by removal of the thymus gland. Br J Surg 1946; 32:201-214

27 Gronseth GS, Barohn RJ. Practice parameter: thymectomy for autoimmune myasthenia gravis (an evidence-based review): report of the Quality Standards Subcommittee of the American Academy of Neurology. Neurology 2000;55(01):7-15

28 Sanders DB, El-Salem K, Massey JM, McConville J, Vincent A. Clinical aspects of MuSK antibody positive seronegative MG. Neurology 2003;60(12):1978-1980

29 Sauerbruch H, Schumacher CB, Roth J. Thymectomy in case of disease with myasthenia. Mitteil Grenzgeb Med Chir. 1913; 25:746

30 Blalock A, Mason MF, Morgan HJ, Riven SS. Myasthenia gravis and tumors of the thymic region. Ann Surg 1939;110(04):544-561

31 Keynes G. The results of thymectomy in myasthenia gravis. BMJ 1949;2(4628):611-616

32 Buckingham JM, Howard FM Jr, Bernatz PE, et al. The value of thymectomy in myasthenia gravis: a computer-assisted matched study. Ann Surg 1976;184(04):453-458

33 Masaoka A, Yamakawa Y, Niwa H, et al. Extended thymectomy for myasthenia gravis patients: a 20-year review. Ann Thorac Surg 1996;62(03):853-859

34 Maggi G, Casadio C, Cavallo A, Cianci R, Molinatti M, Ruffini E. Thymectomy in myasthenia gravis. Results of 662 cases operated upon in 15 years. Eur J Cardiothorac Surg 1989;3(06):504-509, discussion 510-511

35 Emeryk B, Strugalska MH. Evaluation of results of thymectomy in myasthenia gravis. J Neurol 1976;211(02):155-168

36 Calhoun RF, Ritter JH, Guthrie TJ, et al. Results of transcervical thymectomy for myasthenia gravis in 100 consecutive patients. Ann Surg 1999;230(04):555-559, discussion 559-561

37 Pêgo-Fernandes PM, de Campos JR, Jatene FB, Marchiori P, Suso FV, de Oliveira SA. Thymectomy by partial sternotomy for the treatment of myasthenia gravis. Ann Thorac Surg 2002;74(01): 204-208

38 Kas J, Kiss D, Simon V, Svastics E, Major L, Szobor A. Decade-long experience with surgical therapy of myasthenia gravis: early complications of 324 transsternal thymectomies. Ann Thorac Surg 2001;72(05):1691-1697

39 Shrager JB, Nathan D, Brinster CJ, et al. Outcomes after 151 extended transcervical thymectomies for myasthenia gravis. Ann Thorac Surg 2006;82(05):1863-1869

40 Meacci E, Cesario A, Margaritora S, et al. Thymectomy in myasthenia gravis via original video-assisted infra-mammary cosmetic incision and median sternotomy: long-term results in 180 patients. Eur J Cardiothorac Surg 2009;35(06):1063-1069, discussion 1069

41 Agasthian T, Lin SJ. Clinical outcome of video-assisted thymectomy for myasthenia gravis and thymoma. Asian Cardiovasc Thorac Ann 2010;18(03):234-239

42 Gronseth GS, Barohn RJ. Thymectomy for Myasthenia Gravis. Curr Treat Options Neurol 2002;4(03):203-209

43 Skeie GO, Apostolski S, Evoli A, et al; European Federation of Neurological Societies. Guidelines for treatment of autoimmune neuromuscular transmission disorders. Eur J Neurol 2010;17(07): 893-902

44 Liu CW, Luo M, Mei JD, et al. Perioperative and long-term outcome of thymectomy for myasthenia gravis: comparison of surgical approaches and prognostic analysis. Chin Med J (Engl) 2013;126(01):34-40

45 Tomulescu V, Ion V, Kosa A, Sgarbura O, Popescu I. Thoracoscopic thymectomy mid-term results. Ann Thorac Surg 2006;82(03): 1003-1007 
$46 \mathrm{Ng}$ CS, Wan IY, Yim AP. Video-assisted thoracic surgery thymectomy: the better approach. Ann Thorac Surg 2010;89(06): S2135-S2141

47 Yu L, Zhang XJ, Ma S, Li F, Zhang YF. Thoracoscopic thymectomy for myasthenia gravis with and without thymoma: a single-center experience. Ann Thorac Surg 2012;93(01):240-244

48 Ponseti JM, Gamez J, Vilallonga R, et al. Influence of ectopic thymic tissue on clinical outcome following extended thymectomy in generalized seropositive nonthymomatous myasthenia gravis. Eur J Cardiothorac Surg 2008;34(05):1062-1067

49 Ambrogi V, Mineo TC. Active ectopic thymus predicts poor outcome after thymectomy in class III myasthenia gravis. J Thorac Cardiovasc Surg 2012;143(03):601-606

50 Zahid I, Sharif S, Routledge T, Scarci M. Video-assisted thoracoscopic surgery or transsternal thymectomy in the treatment of myasthenia gravis? Interact Cardiovasc Thorac Surg 2011;12(01):40-46

51 Rowse PG, Roden AC, Corl FM, et al. Minimally invasive thymectomy: the Mayo Clinic experience. Ann Cardiothorac Surg 2015;4 (06):519-526
52 Liu Z, Yang J, Lin L, Huang J, Jiang G. Unilateral video-assisted thoracoscopic extended thymectomy offers long-term outcomes equivalent to that of the bilateral approach in the treatment of non-thymomatous myasthenia gravis. Interact Cardiovasc Thorac Surg 2015;21(05):610-615

53 Hess NR, Sarkaria IS, Pennathur A, Levy RM, Christie NA Luketich JD. Minimally invasive versus open thymectomy: a systematic review of surgical techniques, patient demographics, and perioperative outcomes. Ann Cardiothorac Surg 2016;5 (01):1-9

54 Wolfe GI, Kaminski HJ, Aban IB, et al; MGTX Study Group. Randomized trial of thymectomy in myasthenia gravis. N Engl J Med 2016;375(06):511-522

55 Orsini B, Santelmo N, Pages PB, et al; EPITHOR project (French Society of Thoracic and Cardiovascular surgery) Comparative study for surgical management of thymectomy for non-thymomatous myasthenia gravis from the French national database EPITHOR. Eur J Cardiothorac Surg 2016;50(03):418-422 